\title{
Zur Aktionsart in den finnisch-ugrischen Sprachen
}

I. In den meisten fi.-ugr. Sprachen ist weder die Aktionsart noch der Aspekt besonders aktuell gewesen, weil es in der fi.-ugr. Sprachfamilie keine speziellen uralten Ausdrucksmittel (morphologische bzw. syntaktische) für diese sprachlichen Kategorien gibt. Einzelsprachlich sind gewisse Mittel für die Wiedergabe des Aspelits entstanden (so z.B. kann man in einigen ostseefinnischen Sprachen verschiedene Aspekte mit Hilfe des Total- bzw. Partialobjekts ausdrücken, im Weps. dient das aus dem Russischen entlehnte Verbalpräfix do- in einigem Umfang zum Ausdruck des perfektiv-terminativen Aspekts), im Ungarischen sind von altersher genuine Verbalpräfixe (el- 'weg-', meg- u.dgl.) und im Livischen die aus dem Lettischen entlehnten Verbalpräfixe zur Wiedergabe der Aktionsarten gebraucht worden, usw. Gewöhnlich wird die Altionsart, wie u.a. E. ITKONEn in seinem Werk Kieli ja sen tutkimus S. 247-8, 277-8, wo er die Begriffe Aktionsart (fi. tekemislaatu) und Aspekt vom fi.-ugr. Standpunkt definiert, auf Grund der finnischen Beispiele gezeigt hat, mit Hilfe der frequentativ-kontinuitiven und momentanen Verbalsuffixe und deren "Unterarten" ausgedrückt. In seiner Sprachlehre Suomen kielioppi ${ }^{2}$ S. 533-4 (1963) spricht auch A. Pextrilä über die fi. Aktionsart im Zusammenhang mit der verbalen Ableitung. Er stützt sich dabei auf Knut Cannelin, der in Suomi V: 10 S. 17-35 die Aktionsart der denominalen fi. Verbalableitungen behandelt und für diese Aktionsart für das Finnische den Fachausdruck teonlaatu vorgeschlagen hat. CAxvelin seinerseits hat seinen Standpunkt Ajolf Noreens Vårt språk 5. B. S. 607645 entnommen, wo die Aktionsart der Verbalableitungen im Schwedischen eingehend behandelt worden ist. Die fi. Linguisten 
haben also ihre Auffassung von der Aktionsart von Noreex geerbt. Wenn die folgende Stellungnahme gerechtfertigt ist, dürfte diese Auffassung einseitig sein. Die fi.-ugr. Aktionsart kann sich wahrscheinlich auch ausserhalb der Verbalableitung offenbaren.

II. Eine Neuigkeit dürfte zwar für die Linguisten, die vom Standpunkt der allgemeinen Sprachwissenschaft für das Problem der Aktionsart Interesse haben, sein, dass man in der sowjetischen Finnougristik kürzlich die Frage der Aktionsart in gewissen östlichen fi.-ugr. Sprachen, die durch die sog. gepaarten Verben ausgedrückt wird, zu diskutieren angefangen hat. Da die betreffende Literatur für die meisten westlichen Linguisten schwer zugänglich ist, dürfte es sich lohnen, weitere Linguistenkreise hier darauf aufmerksam zu machen. Zugleich wird Unterz. dabei Gelegenheit haben, in einigen diesbezüglichen Teilproblemen seine persönliche Stellungnahme vorzubringen. Nach gewissen sowjetischen Forschern handelt es sich um eine besondere derartige bisher unbekannte Aktionsart im Tscheremissischen, Wotjakischen und vielleicht auch Mordwinischen. U. E. gibt es Spuren davon auch im Syrj. und evtl. auch in den ostseefi. Sprachen, die wir in diesem Zusammenhang gleichfalls heranziehen werden.

Es handelt sich erstens um die Aktionsart im Tscher. und Wotj., die durch die Konstruktion der sogen. gepaarten Verben (russ. sparennyje glagoly) ausgedrückt wird und in der Literatur von den sowjetischen Linguisten M. P. ChaIdze (TbilisiTiflis) und B. A. Serebrennikov theoretisch erörtert worden ist. An der Erforschung dieses Problems hat insbesondere CHaIdze verdienstvoll gearbeitet in seiner Abhandlung Sparennyje glagoly v marijskom jazyke (Joškar-Ola 1960) und kürzlich in dem Aufsatze O proischoždenii i funkcijach marijskich i udmurtskich sparennych glagolov (Über den Ursprung und die Funktion der tscher. und wotj. gepaarten Verben) in Voprosy finno-ugorskogo jazykoznanija IV S. 247-259 (Iževsk 1967). Wenn wir uns auf die letzterwähnte kurz und klar formulierte

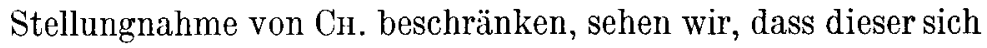
über das zu besprechende Mittel zum Ausdrück der Aktion folgenderweise äussert: "Der gepaarte Verbenkomplex besteht 
(wenigstens) aus zwei Elementen, von denen das erste Verb, das in Gestalt eines Gerundiums auftritt, als das eigentliche Ausdrucksmittel der Handlung fungiert, das zweite aber die Funktion des Präzisierers, Konkretisators der Handlung hat, die von dem ersten Verb ausgedrückt wird. Zusammen drücken die gepaarten Verben die Vorgehensart der Handlung (Aktionsart) aus. Das konkretisierende Verb (das im Falle der nicht inversierten Wortfügung rechts von dem Hauptverb steht) erfüllt dieselbe Funktion, die ein Affix in den präfigierenden Sprachen (z.B. im Russ.) innehat, ausser der Ausdrucksfunktion des terminativen und kursiven Aspekts. Dies bedeutet also, dass sich hier eine neue, bisher unbekannte Art der Wiedergabe der lexikalisch-grammatikalischen Funktionen offenbart, die in den präfigierenden Sprachen Affixen anvertraut ist» (CH. S. 258). Zur Stütze seiner Theorie führt Chaidze S. 250 -257 fünfzehn Beispiele von Modellen der Konstruktionen mit gepaarten Verben an aus dem Wotj. und Tscher. und aus verschiedenen östlichen Sprachen (aus dem Tschuwaschischen, Baschkirischen, Tatarischen, Kazachischen, Mongolischen, Burjatischen, Kalmückischen, Neuchinesischen und dem Hindi), die laut ihm eine gleiche Verbenpaarung aufweisen sollen. Wir möchten hier nur einige wotj. und tscher. Beispiele aus dem Material, das $\mathrm{CH}$. vorgelegt hat, herausgreifen, um die Sachlage in diesen Sprachen zu illustrieren: wotj. lobdźysa lyktyny, tscher. čonešten $(-\eta-)$ tolaš wörtlich 'fliegend (her-, an-)kommen', russ. 'letaja prijti', eigentlich in der Bedeutung '(her-, an-) fliegen'; wotj. lobd'źysa koškyny, tscher. čonešten (- $\eta$-) tolaš wörtl. 'fliegend weg-, fortgehen', russ. 'letaja ujti', in der Bed. 'weg-, fortfliegen', wotj. lobd'źysa džutskyny, tscher. čongešten kuźaš wörtl. 'sich fliegend erheben, fliegend aufsteigen', russ. 'vzletet', in der Bed. 'auffliegen (von der Erde in die Luft)'; wotj. byźysa vuyny, tscher. kuržyn tolaš wörtl. 'laufend (her-)kommen', russ. 'begaja prijti', in der Bed. 'her-, hinzulaufen'; wotj. gožjasa bydtyny, tscher. vozen pytaraš wörtl. 'schreibend abschliessen, beendigen', russ. 'dopisat', in der Bed. 'bis zu Ende, fertig schreiben'; wotj. kyrdźasa leźyny, tscher. muralten koltaš wörtl. 'singend senden, lassen', russ. 'raspevaja pustit', in der Bed. 'zu singen anfangen usw.'; wotj. veraśkysa syli, tscher. 
kutyren šogen (šơen) wörtl. 'sprechend stand', russ. 'beseduja stojal', in der Bed. 'sprach (mit jemandem eine längere Zeit)', usw. Ein Teil der gepaarten Verbkomplexe enthält als Konkretisator ein Verb, das anf längere Dauer der 'Tätigkeit ('stehen', 'leben' usw.) hindeutet, im anderen Teil der Verbalkonstruktionen treten dagegen Verben auf, die auf die Dauer der Tätigkeit nicht Bezug nehmen ('schliessen, beendigen' u.dgl.), aber' diese Gegenüberstellung ist nach (ir. noch nicht rein aspektisch, sondern "voraspektisch", hier handelt es sich nicht um Aspekt, sondern um Aktionsart. Die Entwicklung soll, insbesondere im Tscher. und in den türkischen Sprachen, in der Richtung der Bildung des kursiven und terminativen Aspekts gehen. Um Aspekt handle es sich schon in den Fällen, wo der sog. Konkretisator seine ursprüngliche Bedeutung verloren hat, ein "leerer" (bedeutungsloser) Konkretisator geworden ist (z.B. terminativer Aspekt im Falle tscher. muralten koltas und kursiver Aspekt in solchen Fällen wie tscher. liutyren šogen). S.(H.l.c. S. 253255.

Auf die von Cr. aus den "östlichen" Sprachen vorgelegten Parallelen können wir hier nicht eingehen. Am interessantesten von diesen sind die türkischen Parallelen, die den wotj. und tscher. Konstruktionen sehr nahe stehen. Nicht unbegründet scheint deshalb die Annahme des Verfassers S. 257-8 zu sein, dass die in Frage stehenden wotj. und tscher. Konstruktionen, insbesondere da sie in den übrigen fi.-ugr. sprachen nicht vorkommen, türkische (in erster Linie tatarische und baschkirische) I schnübersetzungen bzw. Calques sein sollten. Cir. ist der Meinung, dass sie auch in den türkischen Sprachen nicht bodenständig sind, sondern aus einem noch weiter im Osten liegenden sprachlichen Zentrum (evtl. aus dem Chinesischen) zu den 'Türken gelangt sind. Er bestreitet die ı.E. an sich sympathische Annahme des Hindologen V. A. Tschensyscuew, dass die gepaarten Konstruktionen in verschiedenen Sprachgruppen spontan, selbständig entstanden sein könnten. Zur Frage über die Entstehung und den ursprünglichen Ausgangspunkt dieser Konstruktionen wagen wir hier nicht Stellung zu nehmen.

Es sei nur zuletzt erwähnt, dass auch B. A. Serebrennikov in seiner Abhandlung Kategorii vremeni i vida $v$ finno-ugorskich 
jazykach permskoj i volžskoj grupp (1960) S. 206 den türkischen Ursprung dieser fi.-ugr. Konstruktionen angenommen hat. X. I. Issnbaev hat in seiner ausführlichen Rezension des Werkes Sparennye glagoly (1960) ron Cr. im Sammelwerk Voprosy dialektologii i istorii marijskogo jazyka (1964) S. 215 -226 einige Fehler des Verfassers gerügt (z.B. dass er die Grenzen der sogen. gepaarten Verben im Tscher. zu weit gezogen habe, indem er auch manche freie Verbalkonstruktionen als gepaarte Verben aufgefasst habe, die mit derartigen zusammengesetzten Verben nichts zu tun hätten, dass er in diesem Zusammenhang einigen älteren Verfassern folgend immer noch rom Aspekt spreche, wo es, wie der Rezensent zeigt, sich nicht um eigentlichen Aspekt handle usw.). In seinem Aufsatz vom J. 1967 hat Cr. wahrscheinlich auf diese Rezension Rücksicht genommen, da er nun nicht mehr allein vom Aspekt im Wotj. und 'Tscher. spricht, sondern die in Frage stehende Erscheinung dieser Sprachen hauptsächlich als Aktionsart qualifiziert. In der zit. Rezension von I. (S. 216) werden auch nützliche Angaben über die Verfasser gegeben, die unsere Frage früher behandelt haben.

III. Chnidze konstatiert in der Einleitung seines Aufsatzes S. 247, dass in den übrigen fi.-ugr. Sprachen ausser dem Wotj. und Tscher. die zusammengesetzten Verben, die er gepaarte Verben nennt, fehlen. In den Anmerkungen S. 258, Fussn. 1 präzisiert er jedoch diese Stelle mit einer Notiz über das Mordwinische: "Es gibt einige Angaben, die davon sprechen, dass auch in einigen mordwinischen Mundarten dieser Typ der verbalen Zusammensetzungen vorhanden ist. In die mordw. Literatursprachen sind diese jedoch bisher noch nicht eingedrungen und auch in den Mundarten sind sie mangelhaft erforscht." Es ist schwer zu sagen, was der Verf. damit eigentlich gemeint hat. Die evtl. türkischen "gepaarten" Verben von dem wotj.tscher. Typ sind wohl sowohl in den mordw. Schriftsprachen als auch in den Mundarten unbekannt (obgleich man dies hinsichtlich der Mundarten wegen ihrer vorläufigen Unerforschtheit nicht ganz bestimmt behaupten kann), aber wenigstens ein Typ der verbalen Zusammensetzungen, der wahrscheinlich als Ausdruck für Aktionsart dient, ist in der Literatur wohlbe- 
kannt und kommt sowohl in den beiden mordw. Schriftsprachen (erz. und moksch.) als auch in den mordw. Dialekten häufig vor. Es handelt sich in erster Linie um die "gepaarten" Verben mit mordw. tejems, tijems 'machen'1 als Konkretisator, die in diesem Zusammenhang beachtet zu werden verdienen.

Die mit tejems gebildeten erzämordw. Konstruktionen hat u.a. schon M. E. JevsevJev in der ersten Auflage seiner Grammatik Osnovy mordovskoj grammatiki (Moskau 1929) S. 200 kurz erörtert. Da dieses Werk uns nicht zugänglich ist, zitieren wir es nach der im J. 1963 von M. N. KoLJADENkov in Saransk publizierten unveränderten zweiten Auflage S. 182: "Schnelligkeit und Kürze der Handlung werden im Mordw. (der Verf. führt nur erzämordw. Beispiele an; J.Mg.) so ausgedrückt, dass man dem Hauptverb das Hilfsverb tejems 'machen' hinzufügt: aštin-tein 'ich sass etwas, ein wenig, eine Weile' (wörtlich 'ich sass-machte'), jarcyn-tein 'ich ass eilig ein wenig' (wörtlich 'ich ass-machte'), avar'c-teis' 'er weinte ein wenig', sims-tejs dy tuś 'er trank eilig ein wenig und ging weg', ojmseś-tejs', tago tuś 'er ruhte sich etwas aus, ein wenig aus und ging wieder (weg)' (tein, tejś im Imperfekt). Die Konstruktion wird als ein be-

${ }^{1}$ Auch in den skandinavischen Sprachen gibt es eine mit dem Verb 'machen' gebildete Verbalkonstruktion, die stark an die mordw. tejemsKonstruktionen erinnert, vgl. z.B. schwed. göra 'machen' in solchen Fällen wie Debuterade gjorde han redan 1915, Blåste gjorde det, sâ det förslog, Men såg programmet gjorde han $i$ alla fall, Arbeta ( arbetar) gör han som slav usw., s. Ordbok över svenska språket 10. (1929) p. 1729 u. folg., wo man dazu sagt, dass diese mit Vorliebe in der Alltagssprache vorkommende Konstruktion eine nemphatische IJerauslösung" des Hauptverbs angeben solle: »emfatisk utbrytning bestående däri att huvudverbet (med bestämningar) lösryckes ur satsen och sättes, antingen i inf. (numera alltid utan att) 1. (med ngt starkare vard. prägel) i den finita form som satssammanhanget fordrar, i satsens början för att däri starkare framhävas, varpå det framför subj. ersättes med den erforderliga formen av göra...». Wenn man auch diese nordische Erscheinung (wie Doz. TrYggve SKöld uns mitgeteilt hat) nicht mit Aktionsart zu erklären pflegt, könnte es sich auch hier ursprünglich um eine hervorhebende, verstärkende $\Lambda$ ktionsart ("för att... starkare framhävas») gehandelt haben. Vgl. auch den eigentümlichen grammatikalischen Gebrauch von do 'machen' im Englischen. Auf diese Fragen der germ. Sprachgeschichte können wir hier natürlich nicht eingehen. 
sonderer Typ der zusammengesetzten Verben bzw. der "Zwillingsverben» angesehen und im Zusammenhang mit solchen für das Mordw. kennzeichnenden asyndetischen verbalen Zusammensetzungen angeführt wie andy-simdi 'bewirtet, speist bzw. gibt zu essen und zu trinken', wörtlich 'speist-tränkt', orči-kari 'zieht, kleidet sich an', wörtl. 'legt Kleider und Bastschuhe bzw. Schuhe an', tri-vany 'erzieht, zieht auf', wörtl. 'hält auf bzw. erzieht-betreut' u.dgl. S. 181-182. In seinen Kommentaren op. cit. S. 434 fasst der Herausgeber Koljadenkov diese tejems-Konstruktionen anders auf: "Den Aspekt, der das Vorsichgehen der Handlung in kleinem Umfang bezeichnet, bildet die Zusammenfügung eines Verbs mit dem Verb tejems 'machen', z.B. kortams-tejems 'etwas, ein wenig sprechen' (wörtl. 'sprechenmachen', J.Mg.), ojmsems-tejems 'sich etwas, ein wenig ausruhen', kištems-tejems 'etwas, ein wenig, etwas, eine Weile tanzen' (wörtl. 'tanzen-machen', J.Mg.). Das Verb tejems wird zu beliebigen Verben hinzugefügt, es bildet einen Ausdruck für eine dauernde (? kursive; J.Mg.) Handlung, die bei Wenigem vor sich geht.) K. weist auf einen Aufsatz vom J. 1948 hin (Filologičeskie doklady na konferencii po voprosam finno-ugorskoj filologii II, Saransk, S. 22-24), wo el die Konstruktion liortams-tejems behandelt hat, der uns aber leider nicht zugänglich ist. KoLJADENkov ist zu dieser Frage, die inzwischen auch von D. V. Bubrich (1947) und B. A. Serebrennikov (1960) behandelt wurde, in seiner erza- und mokscha-mordw. Satzlehre, Grammatika mordovskich (erzanskogo u mokšanskogo) jazykov II, Sintaksis (Saransk 1954), S. 103-106 zurückgekehrt. Ebenso wie seinerzeit JevsevJev geht auch $\mathrm{K}$. hier aus von dem Vergleich der asyndetischen Verbpaare simdems-andoms $\sim$ andomssimdems 'bewirten', erz. oršat-karśat 'du ziehst Kleider an, kleidest dich an', moksch. ščan-karśan 'ich ziehe mich, kleide mich an' u.dgl., in denen nach ihm die Bedeutung der beiden Bestandteile "verallgemeinert" wird und die eine Zwischenstellung zwischen den Wortzusammensetzungen und den syntaktischen Wortgruppen (Zusammenfügungen) einnehmen. Weiter zu den tejems-Konstruktionen übergehend behauptet er S. 104: "Es kommen Fälle vor, in denen in dem Rahmen des paarigen Verbs das eine von den zusammengefügten Verben seine eigent- 
liche Bedeutung verliert, eine verallgemeinerte Bedeutung annimmt und sich damit in ein grammatikalisches Sprachmittel verwandelt. Mit einer solchen Zusammenfügung wird nicht mehr die verallgemeinerte Bedeutung dieser Verben ausgedrückt, sondern eine grammatikalische Variation eines von diesen Verben: erz. hortams-tejems, moksch. korchtams-tijems 'hinreichend lange etwas, ein wenig sprechen', russ. 'pogovorit' dostatočnoe dl’a togo vreńa' (einzeln: erz. kortams, moksch. korchtams 'sprechen', erz. tejems, moksch. tijems 'machen'), erz. jarsams-tejems, moksch. jarchcams-tijems 'etwas, ein wenig nicht eilend, eine hinreichend lange Zeit essen', russ. 'pokušat ne toroṕaś, dostatočnoe dl'a togo vreńla' (einzeln: erz. jarsams, moksch. jarchcams 'essen', erz. tejems, moksch. tijems 'machen'), erz. ojmsems-tejems, moksch. vajmosems-tijems 'sich etwas, ein wenig, so lange es zur Erholung reicht, ausruhen', russ. 'otdochnut dostatočnoe dl'a podkreplenija sil vreḿa' (einzeln: erz. ojmsems, moksch. vajmosems 'sich ausruhen', erz.tejems, moksch. tijems 'machen'). Wörtlich bedeuten die angeführten Wortfügungen also 'sprechen-machen', 'essen-machen', 'sich ausruhenmachen'." Die beiden Komponenten werden hier ebenso wie die Bestandteile in den asyndetischen Wortfügungen vom Typ simdems-andoms konjugiert: erz. kortan-tejan 'ich werde hinreichend lange ein wenig sprechen', vgl. S. 105 z.B. russ. 'pogovorju', 2. Pers. Sing. Präs. erz. lortat-tejat, 3. Pers. Sing. erz. liorty-tei, moksch. lorchtaj-tii, 3. Pers. Sing. Imperf. erz. Son ... ojmaś-tejs 'er ruhte sich ein wenig aus', erz. 3. Pers. Plur. Imperf. aštest-tejst' 'sie sassen ein wenig', russ. 'posideli' usw. Yon dem Typ simdems-andoms unterscheiden sich die tejems-Konstruktionen nach KoLJadexkov dadurch, dass in diesen im Gegensatz zu jenen keine Umstellungen der Bestandteile vorkommen, dass tejems, tijems in ihnen mit einem beliebigen Verb zusammengefügt werden kann, dass sie immer die perfektive Handlung ausdrücken und deshalb nicht im Präsens gebraucht werden und dass sie nicht die Bedeutungen ihrer Bestandteile "verallgemeinern", sondern nur die Bedeutung des ersten Teiles der Zusammensetzung variieren, wodurch das Verb tejems, tijems seine eigentliche Bedeutung verliert und nur dazu dient, im Rahmen des perfektiven Aspekts auf die 
Dauer des ersten Gliedes der Verbalfügung hinzudeuten. Auch die mordwinischen Sprachforscher N. F.Zyganov und S. G. Po's APKIN sollen dem Verf. mitgeteilt haben, dass sowohl in Erz. als im Moksch. die in Frage stehenden Konstruktionen keinesfalls die Kürze der Handlung (wie JevsevJev es aufgefasst hat), sondern im Gegenteil die Handlungen angeben, die während einer längeren Zeit nicht eilend, in Wenigem (bzw. allmählich) ausgeführt werden. Auch Bubricu hat die Sachlage so aufgefasst, dass es sich hier um eine langwierige, dauerhafte Handlung handelt. S. dazu KoLJadenkov op. cit. S. 105 -106. Er ist der Meinung, dass laut dem Gesagten die mordw. tejems-, tijems-Konstruktionen eine "Variation (raznovidnost) des perfektiven Aspektes" bilden (S. 106). Wenn er die Angelegenheit in dieser Weise auffasst, muss man ihn so verstehen, dass er solche formal im Präs. stehenden Fälle (s. oben) wie kortan-tejan, kortat-tejat vielleicht als futurum exachum-Fälle deutet.

Wie wir sehen, ist die semantische Deutung der tejems-Konstruktionen nicht leicht und man hat darüber divergierende Meinungen geäussert. Offenbar handelt es sich, wie KolJadenkov und einige andere mordwinische Forscher vermuten, um eine dauerhafte, in Wenigem (bzw. allmählich) vor sich gehende Handlung, also eine Aktionsart, die an sich nicht perfektiv zu sein braucht. Die Perfektivität ist u.E. hier ein sekundäres Nebenprodukt, das damit zu erklären sein dürfte, dass die tejemsKonstruktionen vorzugsweise im Präteritum (lmperfekt bzw. Perfekt tejś usw.) auftreten. Die dauerhafte, allmähliche $A \mathrm{k}-$ tionsart dürfte hier das Wesentlichste, Primäre gewesen sein. Wie wir schon gesehen haben, können die tejems-Konstruktionen seltener auch im Futur und Präs. auftreten, und es ist vorläufig gar nicht unbestreitbar, dass es sich in solchen Fällen immer um das perfektive futurum exactum handelt. Wir möchten in diesem Zusammenhang auf ein mokschanisches Präsens-Beispiel hinweisen, das aus einer Märchensammlung, also aus der Volkssprache stammt und durchaus authentisch sein dürfte und zwar aus Ustno-poetičeskoe tvorčestvo mordovskogo naroda B. III, 1. Teil, Mokšanskie skazki (Märchen) von A. I. Maskatev, S. 244 Stirś moli bańati $i$ avardi. Avaŕkšni- 
tii, taga moli kulchcondoma, meze korchtai alanc...", vgl. die russ. Übers. S. 251 'Carevna idet (id'jot) $v$ bańu i plačet. Poplačet, pogoŕuet, snora idet podslušivat, o čem (čom) govorit jee (jejo) otec...', dt. 'Die Zarentochter geht in die Sauna und weint. Weint und trauert ein wenig, eine zeitlang, geht wieder um zu horchen, worüber ihr Vater spricht... D Der Kontext erlaubt die in diesem Zitat figurierenden Präsensformen auch als Fälle des praesens historicum aufzufassen. Wenn dem so ist, kann von perfektiven Handlungen die Rede sein. Aber nichts hindert uns, diese Verba im Präsens auch als in den lebendigen Bericht eingeschobene eigentliche Präsentia aufzufassen. Avaŕkšni-tii 'poplačet', 'weint und trauert ein wenig (bzw. einige Zeit)' kann damn kaum ein von Präsensformen umringtes futurum exactum sein, sondern muss auch ein richtiges Präsens mit der Aktionsart 'ein wenig, in Wenigem, einige Zeit' sein und kann in seiner Präsens-Umgebung wohl nicht plötzlich auf den perfektiven Aspekt hindeuten. Die russ. präfigierten Verben auf po- drücken u.E. nicht nur die Perfektivität aus (vgl. 'poplačet'), sondern auch die Aktionsart 'ein wenig', die in unserem Fall wesentlich ist. Da man keine anderen Möglichkeiten hat, die mordw. tejems-Konstruktionen ins Russ. zu übersetzen als mit den perfektiven Präfixverben auf po-, hat man sie mit dem perfektiven Aspekt identifiziert. In der Tat dürfte es richtiger sein, hier von einer alten mordw. Aktionsart zu sprechen, die sich schon im Gemeinmordwinischen vor etwa neunhundert bis tausend Jahren ausgebildet hat, da sie in den beiden mordw. Sprachen (bzw. Hauptdialekten) belegt ist.

Es gibt im Mordw. ausser den eben besprochenen tejemsKonstruktionen noch einige Fälle von zusammengesetzten Verben, in denen mitunter evtl. eine Aktionsart in Frage kommen kann.

Als eine Zusammensetzung von einem Verbalstamm und konjugierten Verb darf man wahrscheinlich folgendes allgemein gebrauchte, einzeln dastehende mordw. Verb ansehen: crz. z.B. nach H. PAasonex, Mordwinische Chrestomathie S. 87 kutmurdams 'umarmen, (einmal) umschlingen', nach dem erz.-r'uss. Wb. von Koljadeykov und N. F. Zyganov S. 116 kutmordams liutmorams 'obnát, obvít', 'umarmen, umschlingen', im moksch.- 
luss. Wb. von S. G. Potapkin und A. K. ImJarekov htmordáms 'obnát (kogo-l.)', '(jemanden) umarmen'. Wir nehmen an, diss dieses Verb aus Paisonen op. cit. S. 86 mordw. kundams 'anfassen, ergreifen; fangen usw.' (auch in den zitierten sowjetischen Wörterbüchern erz. kundams, moksch. kundáms) und PAAsonen op. cit. S. 97 erz. murdams, moksch. mârda.ms 'umkehren, zurückkehren' (in den zit. sowjetischen Wörterbüchern erz. murdams, moksch. midams id.) besteht. Die erste Komponente stellt wahrscheinlich den Verbalstamm kund- $>$ kunt$>$ kut- dar, im zweiten Teil haben wir es wohl mit dem Verb murdams in seiner ursprünglichen Bedeutung 'winden, umwinden, ? wenden' zu tun. Die Zusammensetzung kann ursprünglich die Bedeutung 'greifend umwinden' bzw. 'umwindend greifen' gehabt haben. Wenn diese etymologische Deutung, wio wir glauben, stichhält, liegt hier ein mordw. Einzelfall vor, dessen Bildungsweise der der paarigen tscher. Aktionsartverben sehr nahe steht. Von einer mordw. Aktionsart kann man abel hier nicht sprechen, weil dieses Verbenpaar ganz isoliert dasteht. Vielleicht hat es sich auch hier ursprünglich jedoch um $\mathrm{Ak}$ tionsart gehandelt.

Nach demselben Prinzip Verbalstamm + Verb ist aber im Erzamordw. weiter auch eine Verbalgruppe mit dem Verb PAssones op.cit. 90 merems 'sagen' gebildet. Ein typisches Beispiel dafür findet man bei PAASONEN op.cit. 125 s.r. sịrlist: $s$. merems 'sich ein wenig von der stelle rühren' = sirkstams id. Hier ist sịrkst ein reiner rom Wort sịrgams 'sich in bewegung setzen, aufbrechen' abgeleiteter Verbalstamm. Diese Konstruktion mit merems ist auch in erz. Volksliedern belegt, vgl. z.B. PaAsonex-RAvila MSFOU LXXVII S. 295: 11 sin libort merkšslieśt livtakšnośt' 'auf flogen sie' (vgl. im Wb. von KolJadexkov und Zyganov erz. libordoms 'pórchat', 'schweben, schwirren' und PaAsonex op.cit. S. 90 liviams 'fliegen'), MSFOu LXXVII S. 379: 2 raj libork méran, šiče, mon livtan 'ich fliege auf, Schwager, ich fliege davon', MSFOu LXXXI S. 223: 4 jaliśterk meri pokajse 'mit einem rotschimmernden Festhemd' (vgl. nach KoLJ. und Zya. erz. jakstergadoms 'pokrasnét, zaalét, zarumjánitśa', 'erröten, rot werden' und jaksterdems 'alít, krasnét, rdet', 'rot sein, ins Rötliche spielen'), S. 223: 6 cildork: 
meŕi... meštezę 'leuchtend ist ihr Hemdeinsatz' (vgl. etwa deskr. *cildordoms 'leuchten, schimmern'). In diesen kann u.E. nieŕems, frequ. meŕkšnems als ein semologisch verdunkelter, abstrahierter "Konkretisator" der in der Gestalt der blossen Verbalstämme auftretenden Hauptverben sịrkst, libort, libork, jaks'terk, cildork aufgefasst werden, die sich aber zum Teil wahrscheinlich den mord $w$. Interjektionen auf $-k$ angeschlossen haben.

Als ein semantisch verdunkelter, neutralisierter "Konkretisator" kann im Mordw. mitunter wahrscheinlich auch das Verb PaAsonen MChr. 96 erz. molems, moksch. molams 'gehen, hingehen, hinkommen' fungieren. Man beachte das zusammengesetzte mordw. Verb, erz. nach dem Wb. von KoLJADENkov und Zyganov kaštmolems 'molčát', 'schweigen' und moksch. im Wb. von Potapkix und Imsarekov den Ausdruck kašt af moli 'tišiná, tícho, ni zvúka', 'Stille, Schweigen; still, lautlos' (vgl. Kol.jadenkov und Zyganov kašt 'tišiná', 'Stille, Schweigen' und Interj. a kuš a kaš 'ničegó ne slýšno', 'es ist nichts zu hören, lautlos, geräuschlos'). Mit molems kann vielleicht auch das synonyme erz. Verb PaAsonen MChr. 133 tšatmonems, -muńems, -meńems 'schweigen' gebildet worden sein (evtl. aus einer Interj. tšat- und molems > -mońems, -muńems, -menéms durch Assimilation, evtl. an die Verbalableitungen auf -neangeschlossen?). Aus der im $\mathrm{Wb}$. von KoLJADENKov und ZYGANov belegten Interj. žoj (vom Schellenklang u.dgl.) und molems (im Partiz. -itśa) besteht auch das (partiz.) Attribut im Ausdruck Žoj molitśa pakśań keles 'auf dem wie Schellen klingenden Felde' im erz. Gedichtbuch Eramoś dy škas (Saransk 1962) S. 44 von E. I. PJataev. Wenn man in diesen Beispielen mit molems als "Konkretisator» rechnen kann, ist hier der verbale Inhalt des zusammengesetzten Verbs mit Interjektionen bzw. interjektionalen Substantiven kašt-, tšat'- und žoj ausgedrückt. Im Mordw., wo die Wortklassengrenzen verwischt sind, so dass auch Nomina und bisweilen sogar Interjektionen konjugiert werden können, nimmt dies nicht wunder. Vgl. auch die eben behandelten Konstruktionen mit inerems.

Als einen Fall der paarigen Verben, von denen das zweite 
bedeutungsmässig neutralisiert worden und Suffix geworden ist, behandelt KoLJADExKov op. cit. S. 106-109 den mordw. Konditionalis, vgl. erz. kundynderan $\sim$ kundanderaj, moksch. kundandaran 'wenn ich fange', erz. kundyndarat kundatderaj, moksch. kundand'aŕat 'wenn du fängst' usw. vom Verb kundams 'fangen'. Vgl. PaAsonen op. cit. S. 09 erz. palinídera/n,-t, moksch. palańdera/n,-t 'wenn ich küsse' usw. von palams 'küssen'. Nach K. handelt es sich hier um verbale Zusammensetzungen, in denen ursprünglich die beiden Bestandteile liundams (palams usw.) und *terams bzw. *tarams ( $\left.=t^{\prime} \varepsilon^{-}\right)$'versuchen, streben' konjugiert wurden, später aber die Konjugation, wie man sieht, unregelmässig geworden, gestört worden ist. Diese Erklärung ist nicht ganz sicher, weil das Verb *terams bzw. *'erams im Mordw. nunmehr nicht existiert. Es ist von K. S. 107 nach dem längeren abgeleiteten Verb erz. teraftoms und moksch. taraftoms ( $\left.t^{\prime}-\right)$ 'versuchen' rekonstruiert. Ausserdem gibt es nach K. S. 109 im Moksch. die Partikel d'aŕaj, taraj 'razve, požaluj, koli tak' dt. 'vielleicht usw.', vgl. mon d'araj rianatnen laca 'Ja razve kak snochi', '(mache) ich denn wie die Schwiegertöchter?', Taraj mon molan = mon molind'aran 'wenn ich gehe'. Es bleibt unklar, wie diese Partikel bzw. die Konjunktion, die, wie auch K. einsieht, sicherlich etymologisch mit dem Konditionalsuffix identisch ist, aus diesem entstanden ist. Es ist andererseits nicht ausgeschlossen, dass $d^{\prime} a r^{\prime}-\left(d^{\prime} \varepsilon^{\prime}-\right)$, in dem konjugierten Konditionalis evtl. mit der Endung der dritten Pers. Sing. erweitert (daraj), eine Partikel gewesen ist und als solche Konditionalsuffix bzw. -zeichen geworden ist und als eine konjugierte Partikel zusammen mit dem vorhergehenden Verb eine den paarigen Verben gleiche Konstruktion gebildet hat. Es kann also evtl. gar nicht vom Verbalst. teŕa-, t'ŕra- stammen, sondern ist möglicherweise eine aus dem Tatarischen entlehnte Partikel gewesen. Auf diesen Gedanken führt uns die Tatsache, dass es im Tscher. eine solche entlehnte Partikel gibt, s. z.B. J. G. GrigonJEv Marijskij jazyk (Lehrbuch für Anfänger) S. 83 -84 dyr ( $\delta \delta r$ ) 'verojatno', 'vielleicht, wahrscheinlich', Izat tolynat dyr 'Vielleicht (russ. 'dolžno byt') ist dein Bruder schon gekommen', 15-̌̌e čislalan pyrdyž gazetym luktyda dyr? 'habt ihr die Wandzeitung (vielleicht) bis zum fünfzehnten Tag (des 
Monats) herausgegeben?', zu dieser Partikel $d y$ ' s. auch N. 'I'. Pesigrtov u.a., Sovremennyj marijskij jazyk, Morfologija (Joškar-Ola 1964), S. 305-306. Nach M. RäsÄxex MSFOu L (1923) S. 25 ist dieses $d a r$, tyr 'wahrscheinlich' usw. aus tat. $d a r$, $d y r$ 'vielleicht' entlehnt. Mordw. 'araj (t'-), daraj steht dem tscher. $d y r$ (dar) und dem tat. $d \hat{\partial} r$, dyr so nahe, dass es sich lohnen dürfte nachzuforschen, ob es nicht tat. Ursprungs ist. Wenn es sich wirklich so verhält, kann man beim mordw. Konditionalsuff. natürlich nicht von einem genuinen Verb ausgehen. Dieser Gedanke vom türk. Ursprung des mordw. Konditionalzeichens ist nicht neu. S. auch V. PALL im Autoreferat Vremena i naklonenija $v$ mordorskich jazykach (Tartu 1955) S. 14-15 und Töid läänemeresoome ja volga keelte alalt (Tallinn 196t) S. 56-68, vorsichtig (diese Literaturhinweise verdankt Unterz. Akademiker E. Itkones). Aber da nun eine neue Deutung von KolJADENkov aufgetancht ist, lohnt es sich wohl, die alte Deutung wiederum in Erinnerung zu bringen, wenn auch diese nicht ganz einwandfrei zu sein scheint. Im analytischen mordw. Konditionalis von der Bedeutung 'vielleicht' (<'versuchen') auszugehen ist jedenfalls motivierbar.

IV. Ausser dem Wotj., Tscher. und Mordwinischen begegnet man paarigen Verben bzw. gepaarter Verbzusammenfügung auch noch im Syrj. Mit den obigen Erörterungen bekannt geworden, teilte E. ITKoNEN dem Unterzeichneten brieflich mit (19. 2. 1970): "Im syrj. Wb. von Fokos-Fuchs gibt es ausser den mit den Verben munni und kịlni gebildeten Verbfügungen auch die mit Hilfe des Verbs kerni 'machen' gebildeten, z.T. für das Syrjänische typischen Konstruktionen, deren Anfangsteil man entweder als deskriptiv-onomatopoetische Verbalstämme oder als ebensolche Interjektionen auffassen könnte. Aber es gibt auch einige solche Doppelverben, die man durchaus mit dem mordwinischen Typ avarkšnems-tejems u.dgl. vergleichen kann, z.B. ledźni-kerni 'behexen', verni-kerni 'arbeiten, sich rühren'... Was das Syrj. angeht, kann türkischer Einfluss nicht in Frage kommen."

Diese Beobachtung von ITKones ist zweifellos richtig und wird durch die Angaben gestärkt, die wir in dem unter Redaktion von V. I. LyTkıs herausgegebenen Lehrbuch der syrj. 
Sprache, Sovremennyj komi jazyk I (Syktyvkar 1955) S. 99100 ( $\$ 83 »$ Die deskriptiven phraseologischen Ausdrücke») und S. 249-250 (§ 198 "Deskriptive Verben») vorfinden und die von Lytkis selbst herrühren. Nach ihm (S. 249-250) werden im Syrj. deskriptive Verben u.a. mit den semantisch verdunkelten Verben -kyvny 'gehört werden, hörbar sein', -vid'źny 'sichtbar sein, aussehen', -munny 'gehen' und -kerny 'machen' gebildet, die er als "sekundäre Suffixe" betrachtet. Mitunter werden sie auch mit anderen semantisch "selbständigen» Verben wie vartny 'schlagen' u.a. gebildet. Man beachte z.B. S. 99 raštmuni čegi 'brach krachend' (rašt muni 'Krachen ging, wurde hörbar'), toč-, tač-, tǐ̌-, koč-, kov- munny usw. (von verschiedenen Lauten), kut-, kyr-, vaš-, žuj-, čer-, gyp-vidźny usw. (vom Sitzen, Liegen, Sein in verschiedenen Stellungen), S. 250 Kolnas tup-tapkeris 'Er (sie) stampfte, trampelte mit den Füssen' usw. Der erste Teil dieser Zusammensetzungen, der die onomatop.deskriptive Bedeutung bestimmt, besteht (vorwiegend) aus Interjektionen (vgl. mordw. kaštmolems), der zweite aus irgendeinem semantisch verdunkelten "Konkretisator"), und unter den "Konkretisatoren" findet man ebenso wie im Mordw. u.a. die Verben 'machen' und 'gehen'. Die mordw. und syrj. Konstruktionen haben also manches Gemeinsame, sind aber wahrscheinlich dessen ungeachtet selbständig entstanden, aus gemeinsamen Voraussetzungen der deskriptiv-onomatopoetischen Bewertungsaktionsart hervorgegangen.

V. Wie sich oben herausgestellt hat, haben die paarweise auftretenden asyndetischen Verbalkonstruktionen vom Typ (mordw.) andy-simdi und orči-kari eine Voraussetzung für die Verbpaare geschaffen, die eine Aktionsart wiedergeben. Die asyndetische Zusammenfügung aller Wortklassen, Verben inbegriffen, ist für die finnisch-ugrischen Sprachen kennzeichnend und ist eine uralte Erscheinung in dieser Sprachfamilie, weil sie ursprünglich keine kopulativen Konjunktionen hatte, s. z.B.P. Pulkkinen Asyndeettinen rinnastus suomen kielessä (Helsinki 1966), Einleitung S. 11-18. Wenn wir uns lediglich auf Verben beschränken, können wir uns auf Pulkkinen op.cit. S. 188222 und 291-305 berufen, wo eine ganze Reihe finnische (z.T. auch ostseefinnische, z.B. estn., karel., livische usw.) Beispiele 
für solche Verbpaare vorgelegt werden, z.B. S. 208-210 fi. syövät juozat 'essen (und) trinken', karel. syyväh juuvah id., estn. und liv. süüa juua bzw. siedâ, juodô usw. Die Verbpaare mit den Verben fi. ottaa, estn. võtma 'nehmen', fi. mennä, estn. minema 'gehen' und fi. tulla, estn. tulema 'kommen' als erster Bestandteil, der seine ursprüngliche Bedeutung öfters eingebüsst hat und lediglich als irgendeine Verstärkung des folgenden Teils dient, wie Pulkkinen op. cit. 211--217 konstatiert hat (die "unbalancierten", heterogenen Verbpaare), können u.E. evtl. als Fälle der Aktionsart betrachtet werden. Vgl. fi. Ota-hae wörtlich 'Nimm (und) verschaffe, bring', estn. dial. võtlas, akkas wörtl. 'nahm (und) begann' usw. (weitere Beispiele auch aus den übrigen osfi. Sprachen). Insbesondere die Verben ottaa, võtma und mennä, minema sind hier wahrscheinlich schon semantisch versteinerte Aktionsartmittel geworden. Man beachte auch solche von Istvís PAPP Vir. 1964 S. 130 vorgelegten ung. paarigen Verben wie sütött-fôzzött 'er (sie) briet (und) kochte', eszik-iszik 'er (sie) isst (und) trinkt' usw.

In den osfi. Sprachen gibt es ausserdem einen T'yp von paarigen Verben, den Pulkkinex 1. cit. nicht behandelt hat, der aber erwähnt zu werden verdient, wenn man von der Aktionsart spricht. Dies sind die aus einem beliebigen Verb und irgendwelchem deskriptiven bzw. sonst emphatischen Verb (kolorative Verben nach A. RYTköses) zusammengestellten Verbpaare. Im Finnischen ist diese Konstruktion $u$. E. am reinsten vertreten in solchen Beispielen wie z.B. den von Aarxi PentTrlä, Suomen kielioppi ${ }^{2}$ (Helsinki 1963) S. 566, vorgeführten Fällen Juoksin lönkytin kotiin wörtl. 'ich lief schlenderte nach Hause' = 'ich lief schlendernd' und hän nauroi kihersi puheilleni wörtl. 'er (sie) lachte kicherte zu meinen Worten' = 'lachte kichernd'. Solche Verbalkonstruktionen, die aus den westfi. Mundarten stammen und als zweiten Bestandteil ein mit dem vorgehenden Teil kongruierendes, konjugiertes deskriptiv-onomatopoetisches oder sonst emphatisches Verb enthalten, führt PestTIL $\ddot{A}$ ganz richtig unter "parataktischen Wortzusammenfügungen» an. Auch im Nykysuomen sanakirja I-VI findet man Beispiele dafür, vgl. z.B. I S. 468 s.v. hikertää 'hykertää, kihertää', 'kichern', Kati . . nauroi hikersi sydämessään wörtl. 'Kati 
lachte kicherte in ihrem Herzen' = 'lachte kichernd' u.dgl. Ahti RytKönes, der Vir. 1937 S. 95-100 im Aufsatz "Koloratiivinen konstruktio" Konstruktionen dieses 'Typs behandelt und die Bezeichnung kolorative Konstruktion vorgeschlagen hat für solche Fälle wie z.B. S. 95 purra jauhaa wörtl. 'kauen mahlen', = '(wie) mahlend kauen', S. 96 Työtä tehdä pusertaa wörtl. 'arbeiten drücken' = 'hart arbeiten', Varis lentää vätkistää wörtl. 'die Krähe fliegen schlendert' = 'die Krähe fliegt langsam und plump', S. 97 nauraa hikertää (h. < evtl. schwed. nach RYTköNes) usw., befasst sich, manchmal mit einem unsicheren Resultat, hauptsächlich mit der Etymologisierung der betreffenden »kolorierenden» Verben, ohne darauf Rücksicht zu nehmen, dass die emphatische bzw. onomatopoetisch-deskriptive Art dieser Verben (wenn sie sich auch z.T. als uralte fi.-ugr. Wörter oder ieur. Lehnwörter etymologisieren lassen) massgebend ist. Die von $R$. vorgelegten fi. Beispiele sind meistens in Infinitivformen gegeben, so dass man nicht weiss, wie es sich in diesen Verben mit der Kongruenz der Konjugation in den beiden Bestandteilen verhält. Dies zu wissen ist aber in diesem Zusammenhang wichtig, also ob man es z.B. im Imperfekt mit nauroi hikersi (kongruente finite Formen) oder nauraa hikersi (Infinitiv + Imperfekt) zu tun hat. Die letztere Konstruktion von einer infinitivischen und finiten Verbform ist (z.B. nach $\mathrm{NS}$ ) in der fi. Schriftsprache geläufiger als jene Konstruktion mit zwei kongruierenden parataktischen Verben, vgl. z.B. NS I S. 596 Puhua hölistiin wörtl. 'sprechen plauderten, plauderte man' = 'man sprach plaudernd', IV S. 408 Kattila kiehua porisi wörtl. 'der Kessel kochen brodelte' = 'der Kessel kochte brodelnd', S. 409 Puhua porisee kuin papupata 'er sprechen muckst, brodelt wie ein Erbsentopf' = 'er spricht "brodelnd", ohne Aufenthalt', S. 826 Kukkopoikanen laulaa rämisti 'der junge Hahn krähen klapperte' $=$ '... krähte, schrie mit einer schrillen Stimme'. ${ }^{1}$

1 Weitere Beispiele aus den westfi. Satakunta- und Häme-Dialekten und aus dem Sprachgebrauch des aus dem Kirchspiel Hämeenkyrö stammenden Schriftstellers F. E. SillanP $\ddot{A} \ddot{A}$ hat P. Virtaranta in seinen Arbeiten F. E. Sillanpään puhetta S. 84-85 und Annastuuna aikanansa S. 15'-155 vorgelegt ([Koirra] haukku möllötteli, [poika . . .] 
Im Kar.-Olon., Lüd. und Wepsischen, also in den nächst verwandten osfi. Schwestersprachen des Fi., ist diese kolorative Verbpaarkonstruktion gleichfalls bekannt. Aus dem Karjalan kielen sanakirja von P. VirTarAnta u.a. kann man z.B. folgende kar. und olon. Belege dazu anführen: Suistamo ajau hel(e)hetteä roagieh, Säämäjärvi ajau helhettäy (vgl. helhetteä u.a. 'sich schnell rühren, bewegen'), Suojärvi hebo juoksi helkahutti und kukoi laulaa helkahuttaa (vgl. helkahuttoa 'plötzlich hell klingen usw.'), Rukajärvi matata häipättäy und lunda tulla häipättäy, Suistamo männä häipätti und astuu häipätteä vanhaine (vgl. häipätteä 'schwanken, taumeln'), Säämäjärvi astui häippäi pienil askeluizil 'trat mit kurzen Schritten' und niitti häippäi 'mähte schlecht', Korpiselkä männä häipötteä (vgl. häipötteä 'schwanken, taumeln'), Uhtua pajattoa hyrehtie (vgl. hyrehtie 'hin brummen, summen') usw. Wie man aus den zitierten Beispielen sieht, kommen im Kar.-Olon. sowohl die kongruierenden (astui häippäi usw.) als auch die aus Infin. + konjugiertem Verb bestehenden Konstruktionen (männä häipätti usw.) vor. Im Lüd. scheint der erste von diesen Konstruktionstypen geläufig zu sein, vgl. z.B. nach Lyydiläismurteiden sanakirja von J. KuJoLA nagrau h(orho)ttau (vgl. horhottada 'laut lachen'), duoksou h(örskü)ttä̈̈ (vgl. hörskïttäd̈̈ 'trotten, trottend laufen'), hebod pletia ń(äuk̈äi)żin iškin (vgl. *näukäitä 'schallend schlagen') usw. Im Weps. wird dagegen nach L. KeTrunen, MSFOu LXXXVI S. 153-161 und 490, die Konstruktion von konjugiertem deskr.-onomat. Verb und dem Infinitiv des Hauptverbs ziemlich reichlich gebraucht, vgl. solche Beispiele wie venaks läpätäb baśta 'venättä palpattaa puhua', 'er (sie) plappert russich' (wörtl. 'plappert sprechen russisch'), ka tsu robaidap pagišta 'kas ropistaa haastella', golu śödä mählibad 'aina syödä mähkivät', ruhmip_söda sujas 'hotkii syödä kovasti', paŕzid' jorot' vedada 'hirsiä veti jorotti' usw. Kettunen hält l.cit. (gewöhnlich) das Hauptverb (im Infin.) für ein Adverbiale des

makas lojoili, nauro rötkötti, puhua lässytti u.s.w.). In der Volkssprache dominiert in diesem Gebiet erwartungsgemäss die mit zwei kongruierten Verben gebildete gepaarte Konstruktion, die Konstruktion Infin. + Kolorativverb ist aus der Schriftsprache z.T. in den Sprachgebrauch von SiLla NPä eingedrungen. 
konjugierten deskriptiven Verbs. Die aus zwei kongruierenden, konjugierten Verben bestehenden Konstruktionen scheinen im Weps. selten vorzukommen, also solche Fälle wie nach KETTUNEN op. cit. S. 160 hän riheldab fururdab 'hän viheltää huiluttelee.'

Diese Konstruktion ist auch im Estnischen wohlbekannt, wo sie als ein zusammengesetztes verbales Prädikat angesehen wird, vgl. z.B. K. Kure Eesti keele süntaksi küsimusi (Tallinn 1963) S. 65-66 Kõrt nokitses närida, orast niperdas hõ̃ruda wörtl. 'einen Strohhalm "pickte er kauen", einen Getreidegrashalm "fingerte er reiben", d.h. 'er kaute pickend ..., er rieb fingernd...' Im Estn. (wie auch im Weps.) ist nur die Reihenfolge der verbalen Teile der Konstruktion im Vergleich zu anderen osfi. Sprachen umgekehrt. Vgl. auch estn. ümises laulda wörtl. 'er (sie) brummte singen' = 'er (sie) sang brummend, undeutlich', virises nutta wörtl. 'er' (sie) wimmerte, schwirrte weinen' $=$ 'er (sie) weinte wimmernd, schwirrend' u.dgl. Die zitierten fi. kongruierenden parataktischen Konstruktionen (nauroi kihersi usw.) sind tỵische fi.-ugr. paarige verbale Wortfügungen, die offenbar irgendwelche Aktionsartfunktion haben, obgleich hier der "Konkretisator», Angeber der Aktionsart ( $k i$ hersi, lönkytin), nicht zu einem morphologischen Mittel abstrahiert, semantisch isoliert worden ist wie im Tscher., Wotj., Syrj. und Mordw. (insbesonders in den tejems-Konstruktionen). Eine ganze Verbgruppe, die der onomatopoetisch-deskriptiven und sonst emphatischer Verben, bildet hier u.E. den Konkretisatorkomplex. Die in diesen Konstruktionen zum Vorschein kommende Aktionsart möchten wir die Aktionsart der subjektiven Schätzung bzw. Bewertung nennen. Die behandelten mordw. und vielleicht auch die syrj. Konstruktionen, die onomatopoetisch-deskriptive Elemente enthalten (Typ mordw. kaštmolems, syrj. raštmuni ... usw.) stehen prinzipiell den in Rede stehenden osfi. paarigen Verben nahe. Die aus dem Infinitiv und dem finiten Verb bestehenden osfi. Konstruktionen (nauraa hikersi, puhua porisee, nolitses närida, virises nutta, weps. läpätäb baśta usw.) geben dieselbe Aktionsart an, gehören aber grammatikalisch zu einer anderen Kategorie, zu der des gewöhnlichen zusammengesetzten Prädikats (vgl. Kune op.cit. S. 66 estn. Tuli 
uuesti minna 'es war notwendig, wieder bzw. erneut zu gehen', Vanemad katsusid nooremaid vaigistada 'die Älteren versuchten die Jüngeren zu beschwichtigen' usw.). Es ist möglich, dass der Konstruktionstyp Puhua porisee (im Fi. typisch für die ostfi. Dialekte) und nokitses närida usw. aus der ursprünglichen Konstruktion der paarigen kongruierenden parataktischen Verben puhuu porisee, nokitses näris entstanden ist in Analogie zum zusammengesetzten Prädikat vom Typ tuli uuesti minna u.dgl., der in den osfi. Sprachen allgemein gebraucht wird.

Wir haben bisher zwei ostseefi. Sprachen, das Livische und das Wotische, noch nicht erwähnt, weil uns aus diesen vorläıfig nur spärliche und z.T. unklare Angaben über die »kolorativen" Verbpaarkonstruktionen vorliegen. In den zwei livischen 'Textpublikationen, die wir stichprobenweise studiert haben, in Untersuchung über die livische Sprache von $L$. KetTuses (1925) und Liiviläisiä tekstejä vom Unterz. (1964) sowie auch in Livische Grammatik von SJögREN-WIEdemans (1861) ist es uns nicht gelungen, diesbezügliches Material zu finden. Der gepaarte Konstruktionstyp finites Verb + Infinitiv kommt dort

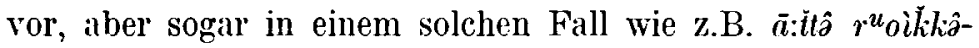
$n \hat{\partial} d \_j \ddot{a} \cdot v \dot{a}$ lä'dô 'sie haben sich beeilt wegzugehen' bzw. 'sie sind eilig weggegangen', Liiv. tekstejä S. 71 (vgl. auch $r^{u}$ oì̀ jùoǩ̌̀ 'beeile dich zu laufen', L. Kettunes Livisches Wörterbuch S. $350^{\mathrm{a}}$, 1938), handelt es sich trotz der affektiven Beschaffenheit des ersten Teiles der Verbfügung schwerlich um eine sichere "kolorative" Konstruktion, die man den oben behandelten estn. und weps. Konstruktionen gleichstellen kann. Es besteht natürlich die theoretische Möglichkeit, dass bei einer genauen Durchkämmung des gesamten gedruckten liv. Materials elwartungsgemäss auch einige sichere Fälle der "kolorativen», die Aktionsart angebenden Konstruktion auftauchen können.

Im Wotischen dagegen sind die Aktionsartkonstruktionen wohl einigermassen im Gebrauch, wie aus den folgenden aus der 'Textsammlung Woten erzählen (MSFOn 118, 1959) vom Verf. geschöpften Beispielen ersichtlich sein dürfte: S. 87 . a u. .lé.kutte:li 'trillerte (und) zwitscherte', S. 27 i viskazi prä·izgütteli 'und [warf,] spritzte (das Wasser)', S. $21 \pm$ IIü·pätti ju:osti po $\cdot$ is 'liefen springend [1. sprangen] davon [weg]', S. 158 r $\ddot{a} \cdot i z-$ 
mitti / rikotti / ne:itě 'zerschmetterte man sie'. Insbesondere das an erster Stelle angeführte wot. Beispiel dürfte den (west)fi., (z.T.) den lyd. und kar. "kolorativen" Aktionsartkonstruktionen genau entsprechen, die aus zwei gepaarten kongruierten finiten Verbformen bestehen. Die übrigen angeführten wot. Verbpaare können evtl. auch als inhaltlich neutrale asyndetischparataktische Verbfügungen aufgefasst werden. Die Lage im Wot. bedarf einer näheren Untersuchung auf Grund eines reichlicheren Belegmaterials.

VI. Wir haben also oben die Rolle der paarigen Verben in der Aktionsart einiger fi.-ugr. Sprachen, und zwar im Wotj., Tscher., Mordw., Syrrj. und in den osfi. Sprachen verfolgt. Die Fragen sind wenig untersucht und deshalb wohl einigermassen strittig, aber eben deshalb haben wir die Aufmerksamkeit auf sie gelenkt, um zu zeigen, dass sie weitere Erforschung verdienen.

Julius MäGiste 